\section{Nachwuchsförderpreis 2008 der DGAKI}

Der Nachwuchsförderpreis der Deutschen Gesellschaft für Allergologie und klinische Immunologie (DGAKI), vormals Herbert-Herxheimer-Förderpreis, gestiftet von der Firma Siemens Medical Solutions Diagnostics $\mathrm{GmbH}$, Bad Nauheim, wird jährlich für herausragende Originalarbeiten verliehen, in diesem Jahr in Erfurt im Rahmen des Dritten Gemeinsamen Deutschen Allergiekongresses, der von 10. bis 13. September 2008 stattfindet.

Der Preis ist zur Auszeichnung besonderer wissenschaftlicher Arbeiten auf dem Gebiet der Allergologie und klinischen Immunologie bestimmt und dient der Förderung jüngerer Wissenschaftler. Zurzeit werden jährlich bis zu $10.000 €$ zu diesem Zweck zur Verfügung gestellt. Der Preis ist für Wissenschaftler aus dem deutschen Sprachraum vorgesehen, die sich noch nicht in Lebensstellung befinden und die das 40. Lebensjahr zum Zeitpunkt der Annahme der Arbeit noch nicht vollendet haben. Ausgeschlossen sind Angestellte von Industriefirmen. Für eine Prämierung kommen Arbeiten in Frage, die sich mit diagnostischen und therapeutischen Fragestellungen oder der Grundlagenforschung auf den Gebieten der Allergologie und klinischen Immunologie sowie verwandter Gebiete befassen.

Die Bewerber reichen bis zum 31. Mai 2008 Originalarbeiten, die bis zu diesem Zeitpunkt publiziert oder zur Publikation angenommen sind, in deutscher oder englischer Sprache ein. Die Arbeiten sollen in fünffacher Ausfertigung vorgelegt werden. Eine Verblindung ist nicht erforderlich. Der Erstautor hat handschriftlich zu versichern, dass er die Arbeit selbstständig erstellt hat. Die Jury kann bei mehr als einer eingereichten preiswürdigen Arbeit den Preis teilen. Je Arbeitsgruppe kann nur eine Arbeit eingereicht werden.

\section{Geschäftsstelle der DGAKI}

Erika Ratzinger, Postfach 7004 64, 81304 München

Tel.: (0 89) 54662968

Fax: (o 89) 583824

E-Mail:dgaki@t-online.de

\section{Förderpreis spezifische Immuntherapie 2008 der DGAKI}

Der Förderpreis spezifische Immuntherapie der Deutschen Gesellschaft für Allergologie und klinische Immunologie (DGAKI), gestiftet von der Firma Bencard Allergie $\mathrm{GmbH}$, München, wird jährlich für herausragende Originalarbeiten verliehen, in diesem Jahr in Erfurt im Rahmen des Dritten Gemeinsamen Deutschen Allergiekongresses, der von 10. bis 13. September 2008 stattfindet.

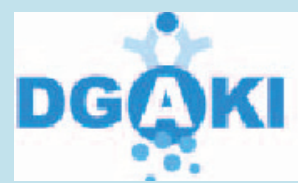

Der Preis ist zur Auszeichnung besonderer wissenschaftlicher Arbeiten auf dem Gebiet der Allergologie und klinischen Immunologie bestimmt und dient der Förderung jüngerer Wissenschaftler. Zurzeit werden jährlich $\mathbf{5 . 0 0 0 € ~ z u ~ d i e s e m ~ Z w e c k ~ z u r ~ V e r f u ̈ g u n g ~ g e s t e l l t . ~}$ Der Preis ist für Wissenschaftler aus dem deutschen Sprachraum vorgesehen, die sich noch nicht in Lebensstellung befinden und die das 40. Lebensjahr zum Zeitpunkt der Annahme der Arbeit noch nicht vollendet haben. Ausgeschlossen sind Angestellte von Industriefirmen. Für eine Prämierung kommen Arbeiten in Frage, die sich mit diagnostischen und therapeutischen Fragestellungen auf dem Gebiet der spezifischen Immuntherapie befassen.

Die Bewerber reichen bis zum 31. Mai 2008 Originalarbeiten, die bis zu diesem Zeitpunkt publiziert oder zur Publikation angenommen sind, in deutscher oder englischer Sprache ein. Die Arbeiten sollen in fünffacher Ausfertigung vorgelegt werden. Eine Verblindung ist nicht erforderlich. Der Erstautor hat handschriftlich zu versichern, dass er die Arbeit selbstständig erstellt hat. Die Jury kann bei mehr als einer eingereichten preiswürdigen Arbeit den Preis teilen. Je Arbeitsgruppe kann nur eine Arbeit eingereicht werden.

\section{Geschäftsstelle der DGAKI}

Erika Ratzinger, Postfach 7004 64, 81304 München

Tel.: (o 89) 54662968

Fax: (o 89) 583824

E-Mail:dgaki@t-online.de

\section{Hufelandpreis für Dermatologen}

$M$ it Festakt im Kölner Gürzenich wurde am 19. März 2008 der Dermatologe Prof. Dr. Swen Malte John, Universität Osnabrück, mit dem Hufeland-Preis ausgezeichnet. Er erhielt den mit $20.000 €$ dotierten Preis für wegweisende Präventionskonzepte für Menschen mit berufsbedingten Hauterkrankungen und Allergien. Der von der Bundesärztekammer und der Bundeszahnärztekammer mitgetragene Preis hat das Ziel, die Präventivmedizin in Deutschland zu fördern. Erstmals seit 1959 geht die Auszeichnung in die Dermatologie.

In Osnabrück werden seit über zehn Jahren berufsdermatologische Präventionsangebote entwickelt, die zu einer erheblichen Senkung der Erkrankungszahlen in verschiedenen Risikoberufen, wie dem Friseurgewerbe und dem Gesundheitssektor, beitragen konnten. Diese Präventionskonzepte werden durch die gesetzliche Unfallversicherung mit Erfolg bundesweit umgesetzt und stehen inzwischen allen Beschäftigten offen: der neue Hautarztbericht, ergänzende ambulante Beratungsmodelle (sekundäre Individualprävention) und das „Osnabrücker Modell“" des stationären Heilverfahrens (tertiäre Individualprävention).

„Vorrangiges Ziel war es, je nach Bedürftigkeit ,passende', aufeinander abgestimmte Präventionsangebote für Menschen mit berufsbedingten Hauterkrankungen und Allergien zu entwickeln, damit alles heute Mögliche unternommen werden kann, um Gesundheit und Arbeitsplatz zu erhalten", erklärte der Preisträger. „Deshalb sind wir auch darüber froh, zurzeit die Nachhaltigkeit der entwickelten Konzepte in bundesweiten Forschungsvorhaben weiter evaluieren zu können."

Um die verbesserten Präventionsstrategien bekannt zu machen, haben die Versicherungsträger die „Präventionskampagne Haut 2007-2008“" ins Leben gerufen. Sie wirbt für einen bewussteren Umgang mit dem größten Organ des Menschen unter dem Slogan „Deine Haut - die wichtigsten $2 \mathrm{~m}^{2}$ Deines Lebens". red 